Research Article

\title{
Locating and Tracking Model for Language Radiation Transmission Based on Neural Network and FAHP
}

\author{
SongGui Zhu, ${ }^{1}$ Hailang He $\mathbb{D}^{2}$ and Yuanyuan $\mathrm{Zheng}^{3}$ \\ ${ }^{1}$ College of Civil and Environmental Engineering, Anhui Xinhua University, Hefei, China \\ ${ }^{2}$ Lanzhou Institute of Seismology, China Earthquake Administration, Lanzhou 730000, China \\ ${ }^{3}$ College of Mechanical and Electrical Engineering, Anhui Jianzhu University, Hefei, China
}

Correspondence should be addressed to Hailang He; hhlmwt@163.com

Received 15 August 2020; Revised 30 September 2020; Accepted 13 October 2020; Published 27 October 2020

Academic Editor: Shaohui Wang

Copyright $\odot 2020$ SongGui Zhu et al. This is an open access article distributed under the Creative Commons Attribution License, which permits unrestricted use, distribution, and reproduction in any medium, provided the original work is properly cited.

With the development of internationalization, the distribution of languages and the office addresses of multinational companies are changing constantly. This paper makes the following research and exploration on this phenomenon: impact on the development of languages around the world. This paper studies the changes of native and second-language users and uses the historical data to predict the development trend by using the gray number series prediction model. Get the types of factors that affect the second language. Then, use fuzzy analytic hierarchy process to calculate the score of each factor. Finally, the global language trend equation is simulated: predictions for the development of language. In this paper, radiation propagation is calculated, and the method of CNN neural network is used to train big data, and the language trend positioning equation is drawn. Finally, the optimal language is obtained by using wavelet analysis and linear programming at different addresses. About model checking, according to the model's internal prediction ability and the significance of internal parameters, it is concluded that the model has high practicability, sensitivity, and stability.

\section{Introduction}

1.1. Background. There are currently about 6900 kinds of languages spoken on Earth. Nearly half of the world's population is using the following ten languages: Mandarin (including Standard Chinese), Spanish, English, Hindi, Arabic, Bengali, Portuguese, Russian, Punjabi, and Japanese [1]. Therefore, these ten languages are called the top ten languages in the world. Much of the world's population can speak the second language. And the development of language tends to be more unified [2].

In the context of economic globalization, many languages are on the brink of extinction [3]. Language is not only a part of culture but also the carrier of culture and the accumulation of traditional culture. If any one language in the world is endangered or even extinct, then the diversity of the world's languages and cultures will be undermined. And it will bring enormous irreparable damage to the colorful language and cultural images of humankind [4].
1.2. Our Tasks. Since a large multinational service company needs the true internationalization, the company proposes to open more international offices, which has already setup offices in New York City in the United States and Shanghai, China.

Here are our tasks:

(1) Determine the influencing factors and the extent of language development. According to the data of the global population and language users over the years, simulate the distribution of users in different languages over time.

(2) Predict the changing trend of the total number of native speakers and language users in the next 50 years. And analyze whether the top 10 languages in the world will be replaced.

(3) According to the established model, predict the global population and population migration patterns in the next 50 years, and determine whether the 
geographical distribution of languages will change in the same period.

(4) Analyze the establishment of six international offices under the identified model. Determine the language in which they are spoken (including English), judging whether there are different impacts in the short and long term.

(5) To save client company resources, analyze whether it is possible to reduce the number of international offices and design management methods.

\section{Assumptions}

By analyzing the article data, we propose the following assumptions to complete our model:

(1) Unpredictable high-impact and low-probability events will not occur in the next 50 years.

(2) For convenience, we assume that a country has only one mother tongue, only the mother tongue or the second-language speaker, and does not consider the third language, etc.

(3) Various languages do not lead to catastrophic jumps in evolution over time.

(4) Except for the factors we considered, other factors have minimal or negligible impact on the model.

(6) Because there are so many languages in the world, we only consider the impact of the main language. Because of its large proportion of the main language, its development can identify the reliability of the model.

\section{Symbol Description and Noun Explanation}

(1) GM $(1,1)$ : a single-sequence line dynamic model in the gray prediction model is mainly used for time series prediction

(2) Train: the data is circulated once

(3) Filter: data-processing weight matrix

(4) Wavelet operation: an efficient algorithm for graphics compression and recognition, which is widely used in various fields where compression of data is required.

\section{The Model}

\subsection{Basic Model (Determination of Influencing Factors)}

4.1.1. Determination of the Influencing Factors. There are mainly two factors that affect the distribution of language users: one is a native speaker and the other is the number and distribution of second-language users [5].

The former is determined by two factors: the number of countries that speak the language as their mother tongue and the population of these countries [6].
The latter is determined by the following factors:

(1) The number of people using the language

(2) The economic strength of the country that uses the language

(3) The literary and social status of the country where the language is used

(4) The degree of importance of language of science and diplomacy (if it is the official language of the United Nations, then we will increase its importance)

For convenience, we depict the following diagram, as shown in Figure 1.

4.1.2. The Proportion of the Influential Factors. Weigh the evaluation criteria in Section 4.1.1, considering the use of fuzzy analytic hierarchy processes [7]. We have the following scoring criteria:

(1) Number of native speakers in this language: highest score 40 .

(2) Number of people who speak the language as their second language: highest score 60 .

(1) The economic strength of the country using that language, with a maximum score of 20

(2) The importance of the language in science and diplomacy, with a maximum score of 15 .

(3) The number of countries and populations using that language, with a maximum score of 15 .

(4) The social and literary status of the language, with a maximum score of 10 .

(5) If the language is for the United Nations, we will add 5 .

The results of the top ten languages in use are shown in Table 1:

4.1.3. The Equation of the Trend of Language Development. The development of future language trends depends on two aspects: native speakers and second-language speakers [8].

The development of future language trends depends on two aspects. The formula is

$$
Y=\lambda_{1} X_{1}+\lambda_{2} X_{2}
$$

In formula (1), the number of native speakers is $X_{1}$ and the number of secondary speakers is $X_{2}$.

(a) The number of native speakers is mainly determined by the changing trend of the population of countries in which the language is the mother tongue. We use the gray number sequence prediction model to describe the language trend. In the gray prediction model, GM $(1,1)$ is a linear dynamic model of firstorder single sequence, which is mainly used for time series prediction. 


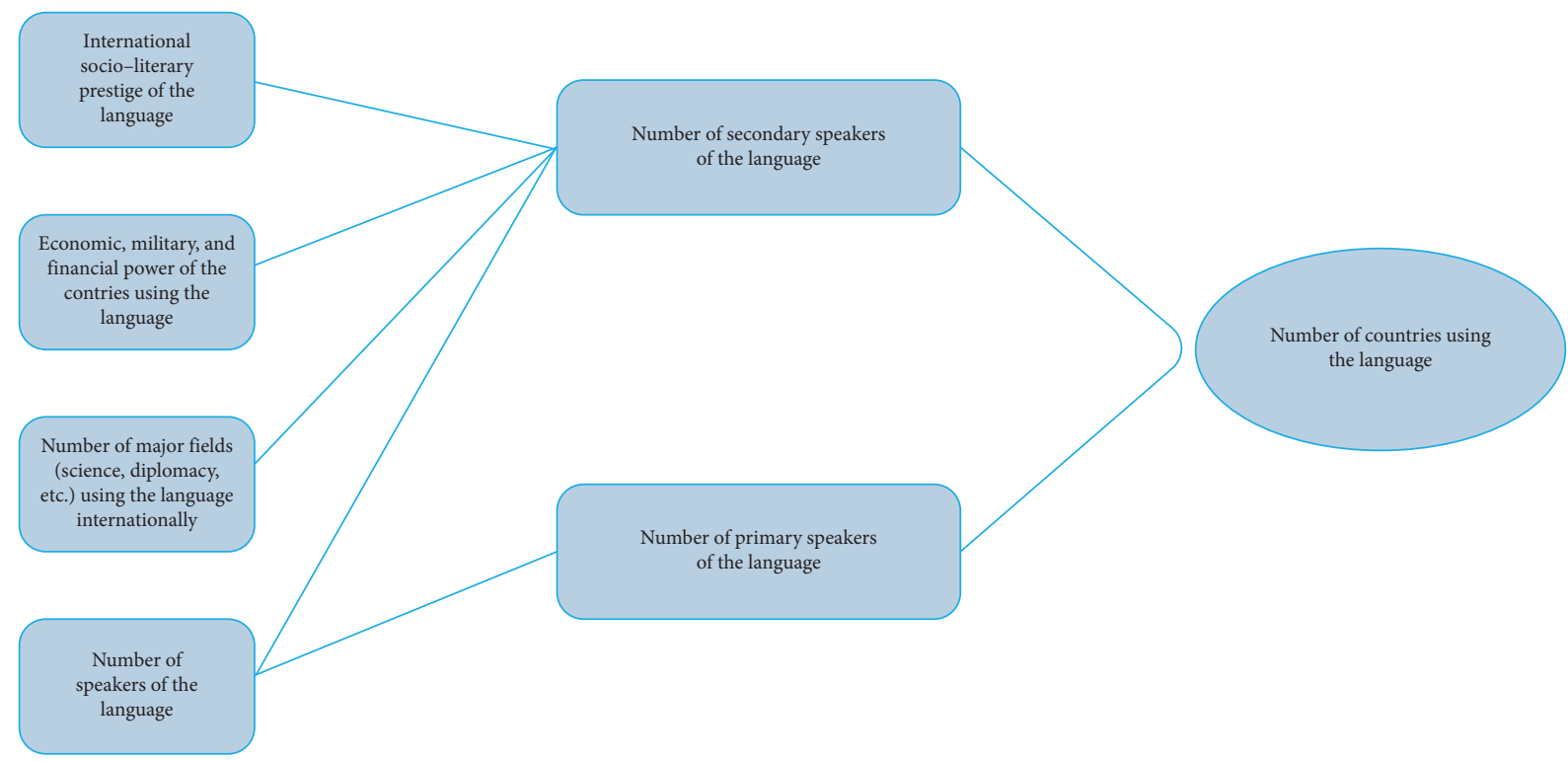

FIGURE 1: Relationship between influential factors.

TABLE 1: The ranking and occupying ratio of language usage.

\begin{tabular}{|c|c|c|c|c|c|c|c|}
\hline Language & $\begin{array}{c}\text { Native } \\
\text { speakers }\end{array}$ & Total & $\begin{array}{c}\text { The proportion } \\
(\%)\end{array}$ & $\begin{array}{c}\text { Second-language } \\
\text { speakers }\end{array}$ & $\begin{array}{l}\text { Number of countries using } \\
\text { the language }\end{array}$ & $\begin{array}{c}\text { The total } \\
\text { weight }(\%)\end{array}$ & $\begin{array}{c}\text { Total } \\
\text { ranking }\end{array}$ \\
\hline Mandarin Chinese & 8.97 & 75 & 11.96 & 1.93 & 10.9 & 14.53 & 1 \\
\hline Spanish & 4.36 & 75 & 5.81 & 0.91 & 5.27 & 7.03 & 2 \\
\hline English & 3.71 & 75 & 4.95 & 6.11 & 9.82 & 13.09 & 3 \\
\hline $\begin{array}{l}\text { Hindustani (Hindi/ } \\
\text { Urdu) }\end{array}$ & 3.29 & 75 & 4.39 & 2.15 & 5.44 & 7.25 & 4 \\
\hline Arabic & 2.9 & 75 & 3.87 & 1.32 & 4.22 & 5.63 & 5 \\
\hline Bengali & 2.42 & 75 & 3.23 & 0.19 & $2 . .61$ & 3.48 & 7 \\
\hline Portuguese & 2.18 & 75 & 2.91 & 0.11 & 2.29 & 3.05 & 6 \\
\hline Russian & 1.53 & 75 & 2.04 & 1.13 & 2.66 & 3.55 & 8 \\
\hline Punjabi & 1.48 & 75 & 1.97 & 0 & 1.48 & 1.97 & 10 \\
\hline Japanese & 1.28 & 75 & 1.71 & 0.01 & 1.29 & 1.72 & 9 \\
\hline
\end{tabular}

Let $\operatorname{GM}(1,1)$ be the original form:

$$
\begin{aligned}
x_{1}^{(0)}(k)+a x_{1}^{(1)}(k) & =b, \\
x_{1}^{(0)} & =\left(x_{1}^{(0)}(1), x_{1}^{(0)}(2), \ldots, x_{1}^{(0)}(n)\right), \\
x_{1}^{(1)} & =\left(x_{1}^{(1)}(1), x_{1}^{(1)}(2), \ldots, x_{1}^{(1)}(n)\right) .
\end{aligned}
$$

Set the basic form of $\operatorname{GM}(1,1)$ to the following formula:

$$
x_{1}^{(0)}(k)+a_{2} z^{(1)}(k)=b,
$$

where

$$
\begin{aligned}
z^{(1)} & =\left(z^{(1)}(2), z^{(1)}(3), \ldots, z^{(1)}(n)\right), \\
z^{(1)}(k) & =\frac{\left(x_{1}^{(1)}(k), x_{1}^{(1)}(k-1)\right)}{2}(k=2,3, \ldots, n) .
\end{aligned}
$$

We define the matrix vector as follows:

$$
\begin{aligned}
& u=\left[\begin{array}{l}
a \\
b
\end{array}\right], \\
& F=\left|\begin{array}{c}
x_{1}^{(0)}(1) \\
x_{1}^{(0)}(2) \\
\ldots \\
x_{1}^{(0)}(n)
\end{array}\right|,
\end{aligned}
$$

$$
B=\left|\begin{array}{cc}
-z^{(1)}(2) & 1 \\
-z^{(1)}(3) & 1 \\
\ldots & \ldots \\
-z^{(1)}(n) & 1
\end{array}\right| .
$$




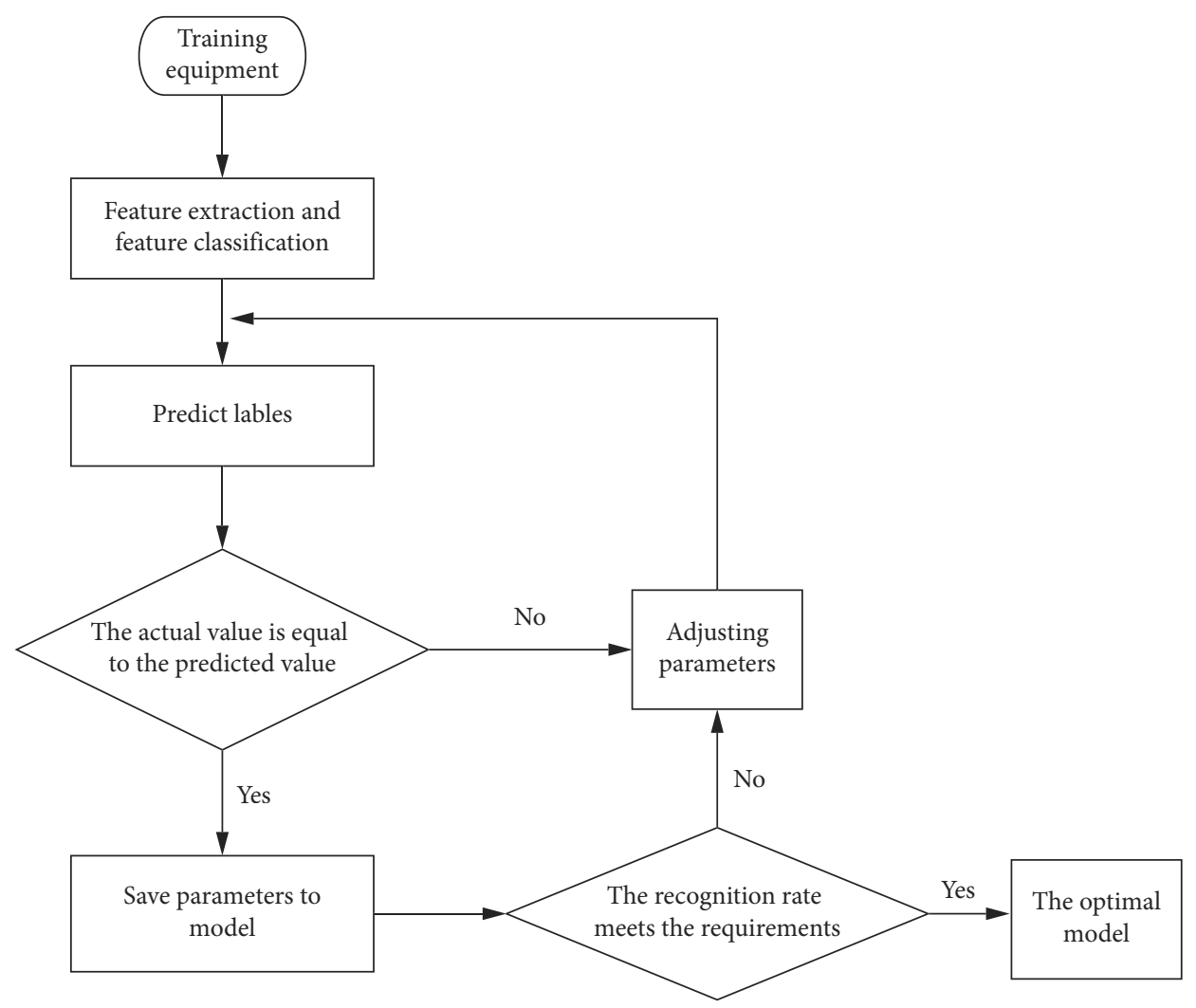

FIGURE 2: Flowchart of CNN neural network.

Putting these data into the above formula, we get $a, b$.

(b) For the second-language trend forecast and from the above factors, we have

$$
x_{2}=\eta x_{1}
$$

Where $x$ is the language synthesis coefficient.

Therefore, from the above formula, we have the language trend equation:

$$
Y=\left(\lambda_{1}+\lambda_{2} \eta\right) F
$$

4.1.4. Accurate Positioning through the Radial Communication of the Global Language. The spread of language is affected by the above factors, but we think the root cause is the radiative propagation of the source language. We use CNN neural network to do big data simulation [9] and depict aflowchart to indicate the exact location of the steps, as shown in Figure 2.

We simulate the distribution, assuming the location coordinates $(x, y)$ and the time factor $t$. Big data simulation is used for filtering to find the specific feature $N$ in the input. Eigenvalues are used for prediction equations and high precision calculations [10].

We have the following equations:

$$
\begin{aligned}
Y(\eta, t, s) & =\frac{\xi}{\sum_{n=0}^{x_{1}-1}\left(\left(\left(x_{2} / t\right)^{n} / n !\right)+\left((\eta / t)^{n} / n !\right)\right)}, \\
\xi & =\frac{1}{x t(1-(\eta / y t))} .
\end{aligned}
$$

\subsection{Application of the Model}

4.2.1. Distribution of Language Users. Based on the global language trend positioning model, as time goes by, due to the variety of languages, here we select only the top 5 languages of the world to simulate the global rough distribution of speakers of this language in the next 50 years. With the office as the point and the contact between each office and the outside world as the degree, the topological relationship can be used for analysis [11].

It is clear from the pictures that the above five languages all have the following development trends:

(1) The population of countries that are native speakers of the language is on the rise

(2) The number of countries in that language as a second language is also on the rise

4.2.2. Future Language Development Trend. According to the change of the number of language users in the first 50 


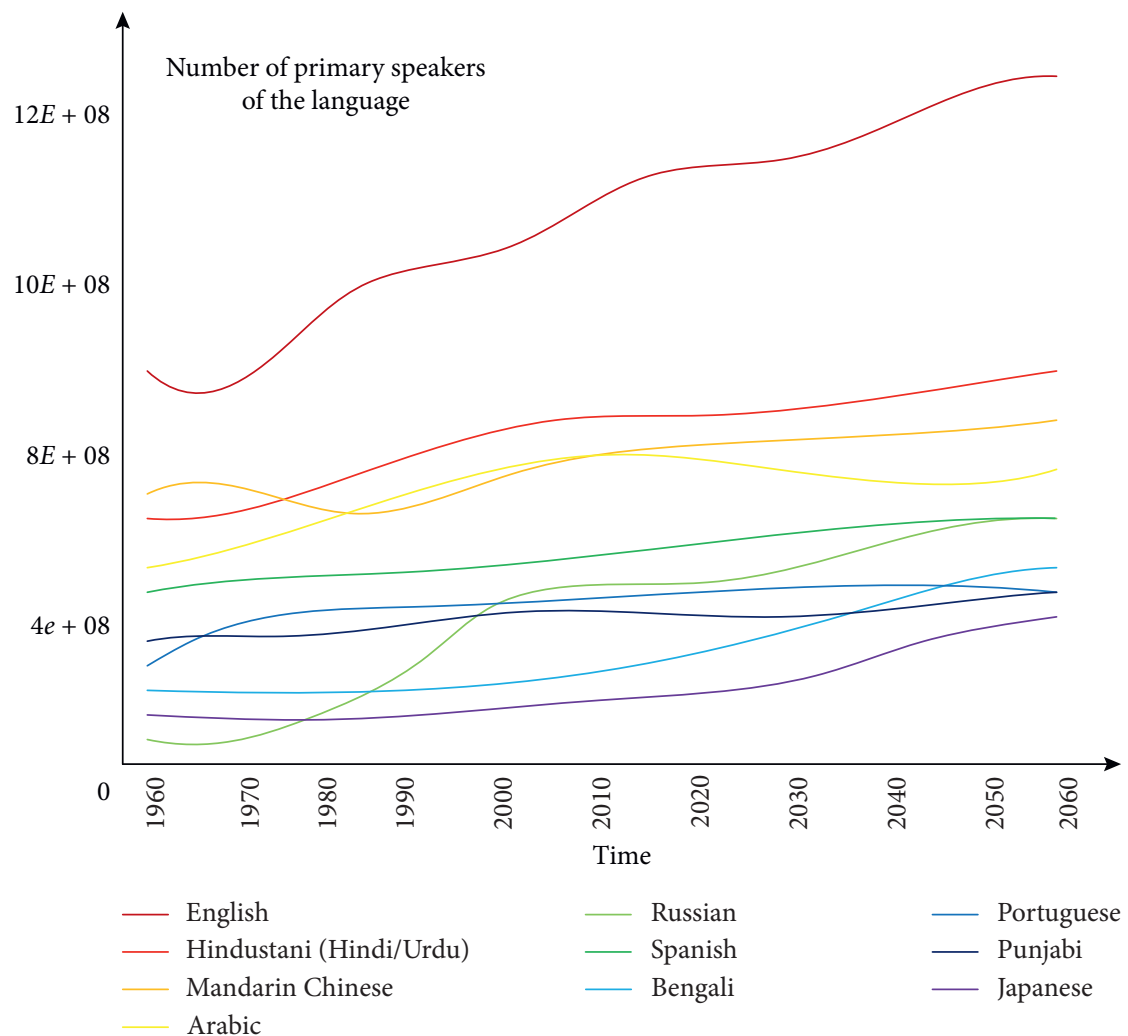

Figure 3: Number of native speakers from 1960 to 2060.

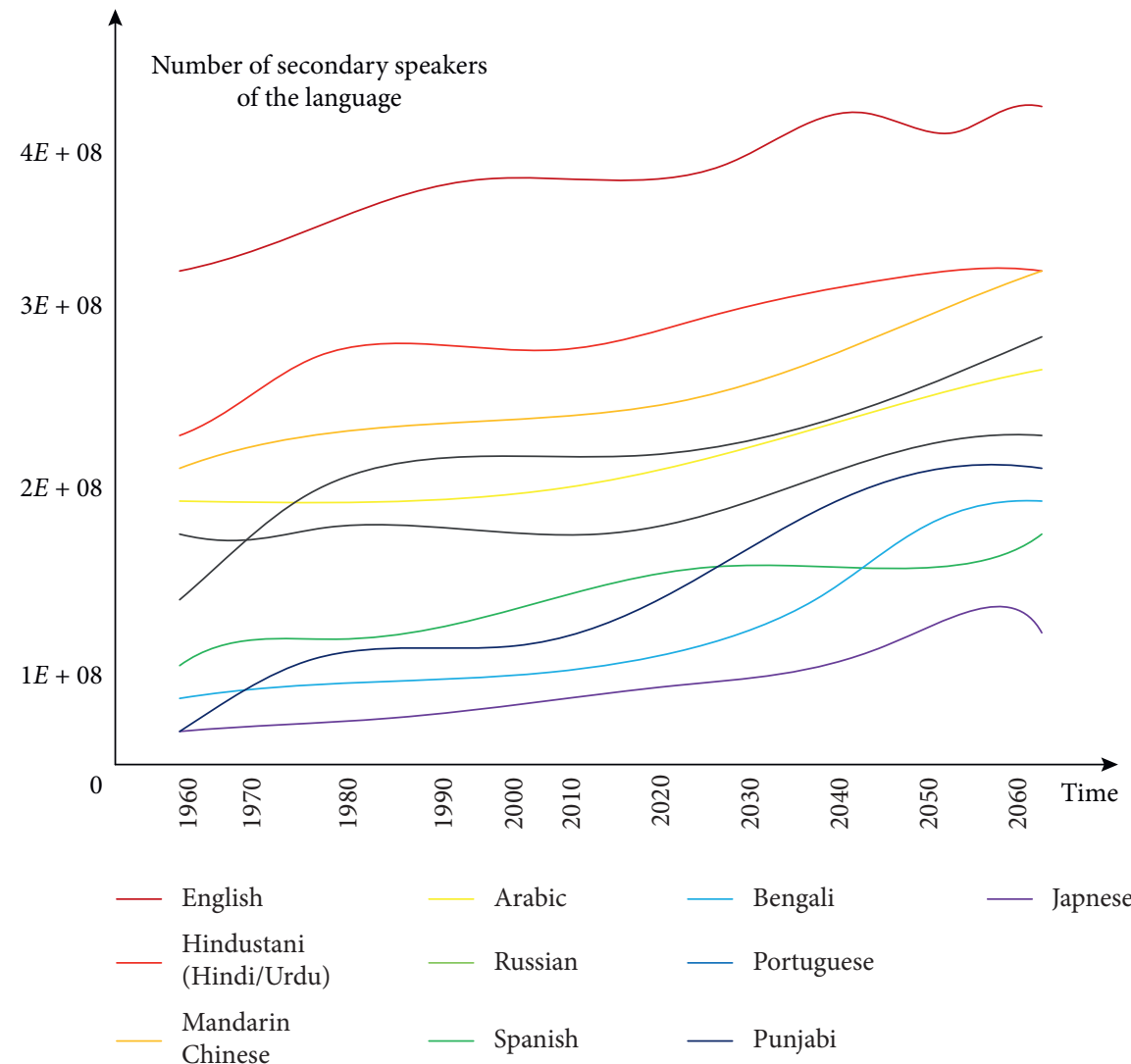

Figure 4: Number of secondary speakers from 1960 to 2060. 


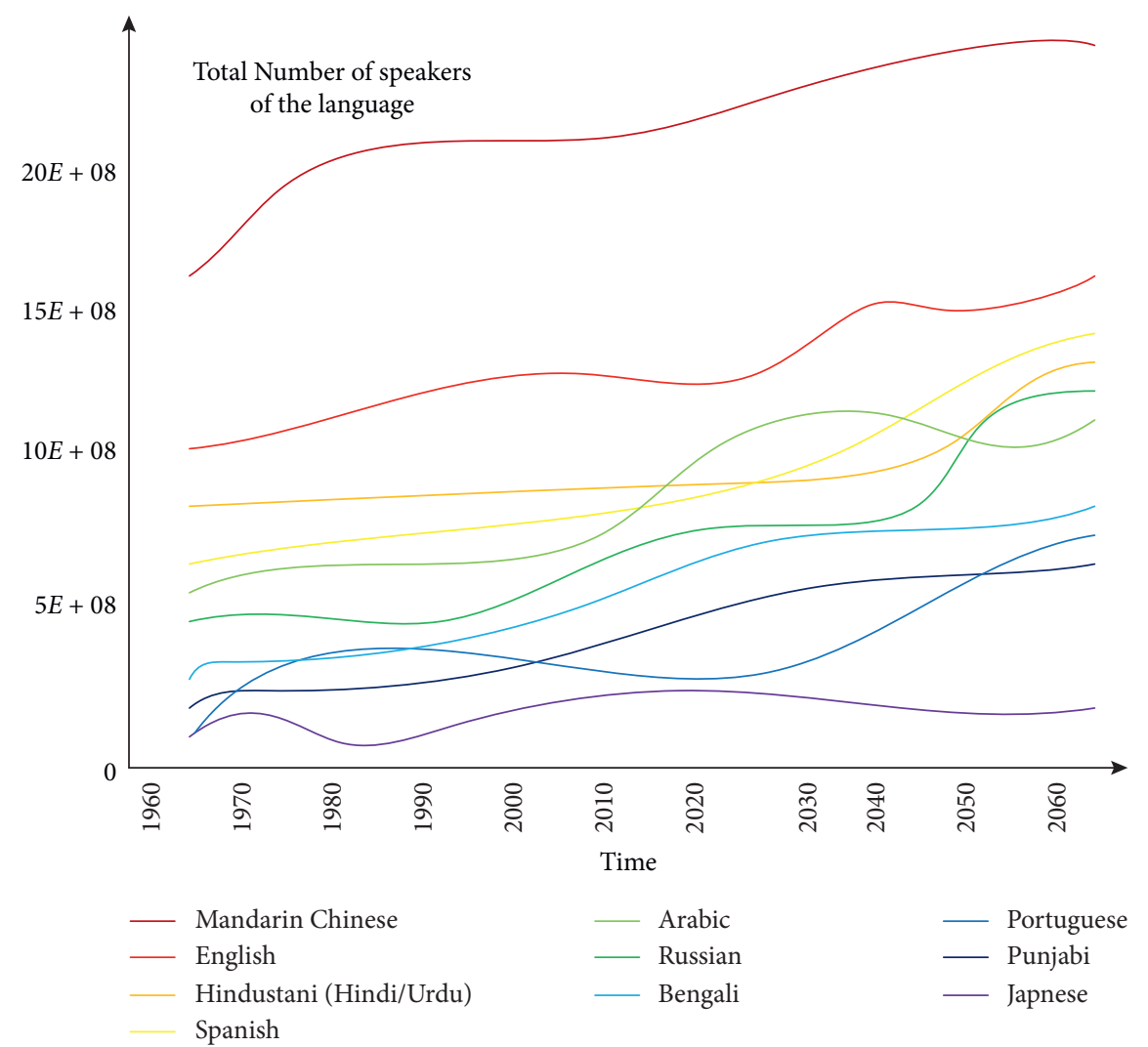

FIgURE 5: Total number of speakers from 1960 to 2060.

years and according to the established formula, the coefficient matrix remains unchanged and the time coefficient increases so that the ratio of coordinate position to trend coefficient will increase significantly. Therefore, the total number of people who speak the language will increase dramatically.

To describe the trend of language development, we depict the following diagram, as shown in Figures 3-5.

Similarly, we can substitute the remaining five languages in Figure 5 to predict the distribution in the same way. By predicting the top ten languages, we think there will be languages in other ten languages of the world that are replaced by other languages. The reason is as follows.

Although the world's total population will eventually stabilize, it can be seen from Figure 5 that the number of speakers with languages continues to increase, great instability will occur in both countries and regions, and the number of second languages will continue to increase. And there is a tendency to approach the number of native speakers. So, anyone of the top ten languages may be replaced.

\section{Sensitivity Analysis}

Based on the internal fitting degree of the model, the significance of the parameters in the model, and the internal and external predictive ability of the model, we established a predictive model with good fit, strong stability, and good predictive ability.
In the first part, we establish a multifactorial global language localization prediction model. Due to the large number of factors, it is not conducive to the verification of the sensitivity of the variable change. Therefore, we use the data internally prediction test, using the data of 1960-2007 global languages as the database. We reanalyzed the languages' comprehensive ability coefficient $\eta$ for that period; putting it into the model we set up, we predicted the situation for the next decade, compared it with the actual total language population for 2007-2017, and plotted it in the same figure to compare, as shown in Figure 6.

The error is

$$
m=\frac{\sum_{i=0}^{n}\left|x_{i}^{\prime} / x_{i}-1\right|}{n} .
$$

Take the same distance 100000 number set to $n$, as shown in Table 2.

As can be seen from the above table, the error $m$ is less than $0.5 \%$, so the model is more closely related to the language trend forecast in the short term.

\subsection{The Advantages of the Model}

(1) Based on the correct analysis of the influencing factors, we use the correct data processing method to solve the multifactor optimization problem well and establish a predictive model with high fitness, good stability, and good predictive ability. 


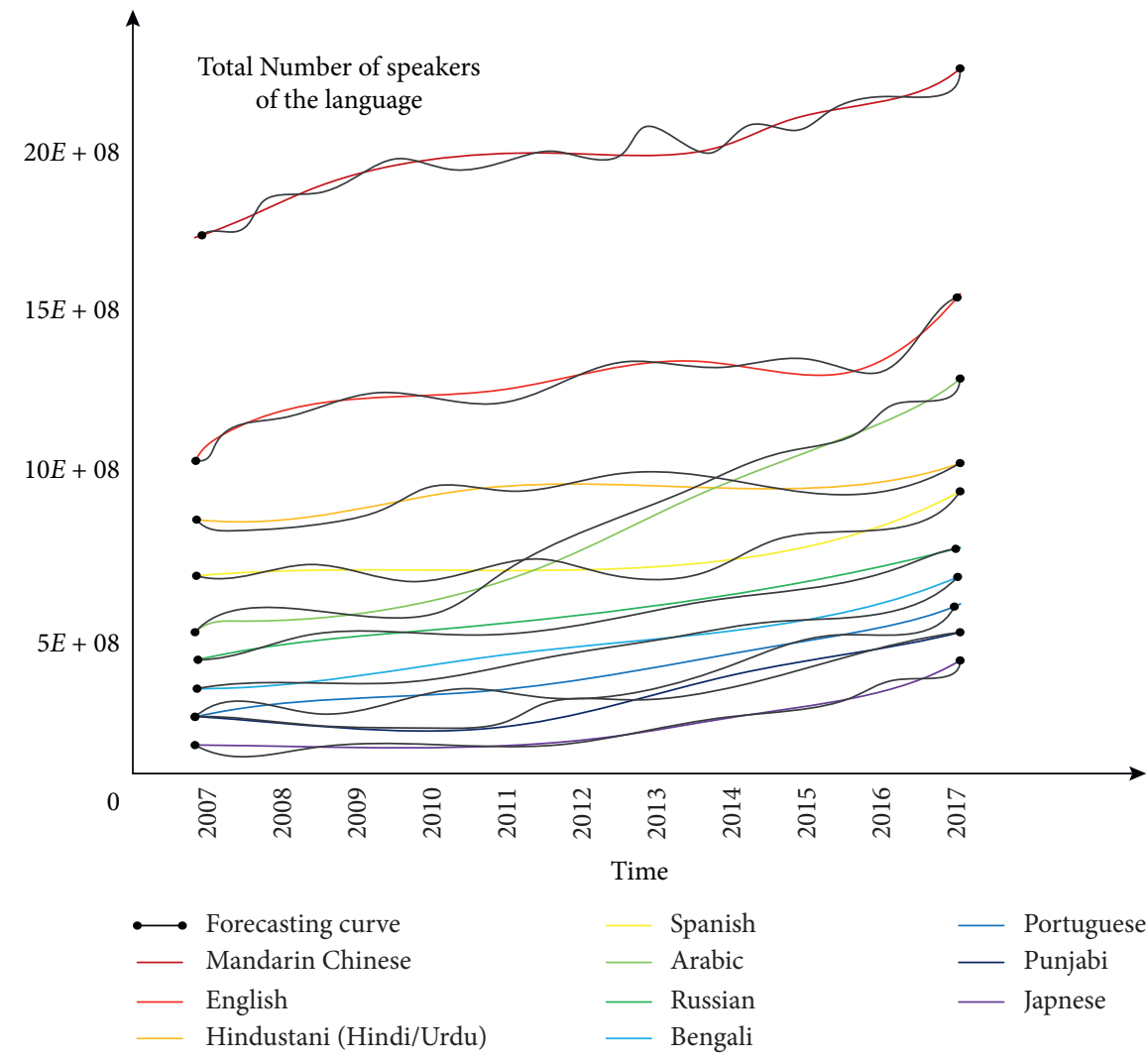

Figure 6: Analysis chart of actual and forecast results.

TABLE 2: Error analysis table.

\begin{tabular}{lcccccccccc}
\hline Languages & Mandarin Chinese & English & Hindustani & Spanish & Arabic & Russian & Bengali & Portuguese & Punjabi & Japanese \\
\hline$M(\%)$ & 0.48 & 0.35 & 0.32 & 0.24 & 0.18 & 0.15 & 0.25 & 0.10 & 0.08 & 0.06 \\
\hline
\end{tabular}

(2) We conduct a sensitivity analysis based on the internal fitting degree of the model, the significance of the parameters in the model, and the internal and external predictive ability of the model. The internal parameters of the prediction test and the fitting test obtained Table 2, and the internal and external parameters of the test of significance test is stable, so our team model has good applicability.

\subsection{The Disadvantages of the Model}

(1) Introducing too many variables in the process of model establishment can easily lead to "dimensionality disaster," which is not conducive to programming

(2) This model has a parameter hypothesis, but it cannot avoid the model deviation caused by some actual factors, which may not be completely in line with the actual situation
Hopefully, the above reference suggestions will be very helpful to the readers.

\section{Data Availability}

The data used to support the findings of the study are available from the corresponding author upon request.

\section{Conflicts of Interest}

The authors declare that they have no conflicts of interest.

\section{Acknowledgments}

This work was supported by Key Projects of Support Program for Outstanding Young Talents in Anhui Province Colleges and Universities (no. gxyq 2018119) and Key Project Funds from Anhui Education Ministry (no. 2019 rcsfjd089). 


\section{References}

[1] W. G. Mitchener, "A stochastic model of language change through social structure and prediction-driven instability," SIAM Journal on Applied Mathematics, vol. 77, no. 6, pp. 2272-2293, 2017.

[2] B. J. Seggewiss, T. Straatmann, K. Hattrup, and K. Mueller, "Testing interactive effects of commitment and perceived change advocacy on change readiness: investigating the social dynamics of organizational change," Journal of Change Management, vol. 19, no. 2, 2019.

[3] D. Sayers, "The mediated innovation model: a framework for researching media influence in language change," Journal of Sociolinguistics, vol. 18, no. 2, pp. 185-212, 2014.

[4] G. Baxter and W. Croft, "Modeling language change across the lifespan: individual trajectories in community change," Language Variation and Change, vol. 28, no. 2, pp. 129-173, 2016.

[5] S. J. Greenhill, C.-H. Wu, X. Hua, M. Dunn, S. C. Levinson, and R. D. Gray, "Evolutionary dynamics of language systems," Proceedings of the National Academy of Sciences, vol. 114, no. 42, pp. 8822-8829, 2017.

[6] J. A. Jódar-Sánchez, "The handbook of historical sociolinguistics," Language in Society, vol. 44, no. 5, 2015.

[7] K. Stadler, R. A. Blythe, K. Smith, and S. Kirby, "Momentum in language change," Language Dynamics and Change, vol. 6, no. 2, pp. 171-198, 2016.

[8] R. Borges, "The role of extralinguistic factors in linguistic variation and contact-induced language change among Suriname's Coppename Kwinti and Ndyuka Maroons," Acta Linguistica Hafniensia, vol. 45, no. 2, pp. 228-246, 2014.

[9] A. D. M. Smith, "Models of language evolution and change," Wiley Interdisciplinary Reviews: Cognitive Science, vol. 5, no. 3, pp. 281-293, 2014.

[10] F. Fu and M. A. Nowak, "Global migration can lead to stronger spatial selection than local migration," Journal of Statistical Physics, vol. 151, no. 3-4, pp. 637-653, 2013.

[11] J.-B. Liu, J. Zhao, H. He, and Z. Shao, "Valency-based topological descriptors and structural property of the generalized sierpiński networks," Journal of Statistical Physics, vol. 177, no. 6, pp. 1131-1147, 2019. 